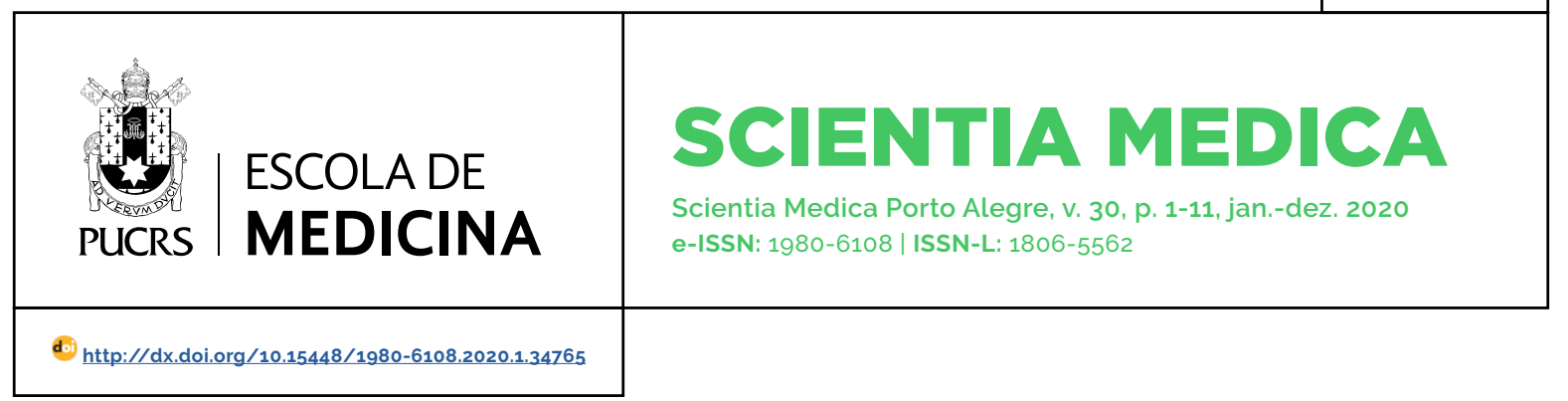

ARTIGO ORIGINAL

\title{
Desenvolvimento e Validação do Questionário de Conhecimento em Asma Pediátrica (Q-CAP) para população brasileira
}

\author{
Development and validation of Knowledge in Pediatric Asthma questionnaire (Q-CAP) \\ for brazilian population
}

\author{
Thiago de Araujo \\ Cardoso ${ }^{1}$ \\ orcid.org/0000-0002-2717-644X \\ profcardoso@live.com
}

Mariele Cunha Ribeiro²

orcid.org/0000-0003-0595-1043

ribeiro_mariele@hotmail.com

\section{Cristian Roncada ${ }^{3}$}

orcid.org/0000-0003-3782-4911

crisron@gmail.com

\section{Rejane Rosaria Grecco dos Santos 4}

orcid.org/0000-0003-0326-0839

rejanegrecco@ymail.com

\section{Paulo Márcio Condessa Pitrez $^{5}$}

orcid.org/0000-0001-7319-1133 ppitrez70@gmail.com

Recebido em: 10 jul. 2019.

Aceito em: 31 out. 2019.

Publicado em: 25 mai. 2020 .

\begin{abstract}
RESUMO
OBJETIVO: Desenvolver e validar o Questionário de Conhecimento em Asma Pediátrica (Q-CAP), na lingua portuguesa brasileira.

MÉTODOS: o questionário foi desenvolvido com base em instrumentos internacionais reconhecidos. Especialistas analisaram o instrumento, juntamente com pais de crianças com asma. Foram realizados os testes de validade de construto e conteúdo, alfa de Cronbach, coeficiente de correlação intraclasse, correlação de Pearson, análise de Kappa e curva ROC.
\end{abstract}

RESULTADOS: participaram do estudo 132 pais de crianças com asma, sendo 117 $(88,6 \%)$ do sexo feminino, idade média de 35,9 $\pm 9,1$ anos, 78 (59,1\%) com ensino médio completo, 73 (59,8\%) pertencente a classe econômica C. A versão final do instrumento possui 20 questões, com ponto de corte de $\geq 14$ pontos. Os escores totais obtiveram conceito "A" nos testes de validade/constructo e valor de 0,69 no alfa de Cronbach. Na avaliação da correlação do coeficiente intraclasse o questionário possui níveis dentro dos aceitáveis (ICC=0,69; IC95\%=0,61 0,77; $\mathrm{p}<0,001$ ). A sensibilidade e especificidade apresentaram área de 0,93 (IC95\%: 0,84 1,0; $p=0,002$ ) e valores de $80 \%$ e 97,1\%, respectivamente. Após a aplicação da versão final do Q-CAP, apenas 5 (3,8\%) dos pais demonstraram niveis satisfatórios de conhecimento em asma.

CONCLUSÃO: O Q-CAP demonstrou ser confiável, claro e com adequados indices de reprodutibilidade e consistência interna. Sua aplicação é fácil e abrange os aspectos culturais brasileiros essenciais, além de grande precisão em mensurar o nivel de conhecimento sobre asma.

PALAVRAS-CHAVES: Asma; conhecimento em asma; questionários; pais; educação em saúde; psicometria.

\section{ABSTRACT}

AIMS: To develop and validate the Knowledge Questionnaire in Pediatric Asthma, in the brazilian Portuguese language.

METHODS: The questionnaire was developed based on recognized international instruments. Experts have examined the instrument, along with parents of children with asthma. The tests of construct validity and contents, Cronbach's alpha, intraclass correlation coefficient, Pearson Correlation, analysis of Kappa and ROC curve.

RESULTS: Participated in this study 132 parents of children with asthma, and 117 (88.6\%) female, average age of $35.9 \pm 9.1$ years, 78 (59.1\%) with full high school, $73(59.8 \%)$ in the economy class $C$. The final version of the instrument possessing 20 questions, with cut-off of $\geq 14$ points. The total scores obtained "A" concept validity tests/construct and value of 0.69 on Cronbach's alpha. In the evaluation of correlation intraclass coefficient the questionnaire has within the acceptable 
levels (ICC =0.69; 95\% Cl=0.61 0.77; $\mathrm{p}<0.001)$. The sensitivity and specificity have lodged area of 0.93 (95\% Cl: $0.84 \sim 1.0 ; \mathrm{p}=0.002$ ) and values of $80 \%$ and $97.1 \%$, respectively. After applying the final version of the questionnaire of knowledge in Pediatric Asthma, only $5(3.8 \%)$ the parents showed satisfactory levels of knowledge in asthma.

CONCLUSION: The Knowledge questionnaire in Pediatric Asthma has proven to be reliable, clear and with suitable levels of reproducibility and internal consistency. Your application is easy and covers the cultural aspects, as well as Brazilian great accuracy in measuring the level of knowledge about asthma.

KEYWORDS: Asthma; Knowledge of asthma; Questionnaires; Parents; Health education; Psychometrics.

ABREVIATURAS: NAKQ, Newcastle Asthma Knowledge Questionnaire; Q-CAP, Questionário de Conhecimento em Asma Pediátrica.

\section{INTRODUÇÃO}

A asma é uma doença respiratória crônica que acomete cerca de 300 milhões de pessoas mundialmente [1]. No Brasil, a prevalência da doença é elevada, com mais de 2.000 mortes registradas só em 2013, isto é, aproximadamente cinco óbitos/dia, e mais de 120.000 hospitalizações no mesmo periodo [2]. Considerando que 20\% das crianças brasileiras possuem diagnóstico da doença e as taxas de hospitalizações e óbitos são altas, é importante que estas tenham acompanhamento médico sistemático a fim de traçar um plano de tratamento customizado, respeitando as particularidades, necessidades e limitações do indivíduo [2, 3].

Em pacientes pediátricos, os pais são os agentes centrais no manejo da doença, sendo responsáveis pela gestão do tratamento e aplicação dos medicamentos. Visto que, o alto nivel de conhecimento sobre a asma foi associado à diminuição da restrição de atividade física, absenteismo escolar, visitas ao serviço de emergência e sibilância noturna, bem como melhora da função pulmonar [4], é imprescindivel que os pais dominem os principais conceitos sobre a doença, tais como: sintomas, gravidade, efeitos dos medicamentos e técnicas inalatórias. Diversos são os meios de comunicação que auxiliam na educação em saúde dos pais e que propiciam o aumento na adesão do tratamento. Mas nem todos são confiáveis, muitos destes fornecem informações erradas e com pouco critério científico, disseminando mitos populares que deturpam a realidade e diminuem a adesão ao tratamento [5].

Para que a equipe de saúde possa mensurar os niveis de conhecimento dos pais e a qualidade das informações adquiridas a aplicação de instrumentos avaliativos durante as consultas se faz necessário. Embora não exista um consenso sobre qual o melhor instrumento a ser utilizado, alguns critérios de qualidade devem ser observados, como a validade do conteúdo/constructo, a reprodutibilidade e a consistência interna [6]. Ao consultar a rede mundial de computadores (internet) diversos questionários com essa finalidade são encontrados [7, 8], entretanto, quase a totalidade destes necessitam de algum tipo de adaptação cultural e/ou linguísticas para que possam se adequar a realidade da população brasileira [9-11]. Essas adaptações, por vezes, acabam por descaracterizar a versão original do instrumento e fragilizam os resultados das análises psicométricas, pondo em risco a confiabilidade dos resultados.

Com base nessa perspectiva, é necessário que um instrumento genuinamente brasileiro, que avalie os niveis de conhecimento em asma de pais de crianças asmáticas, seja desenvolvido e validado, levando em consideração os aspectos culturais e linguísticos da população brasileira, sem a necessidade de traduções e adaptações. Sendo assim, o presente estudo teve como objetivo construir e validar o Questionário de Conhecimento em Asma Pediátrica (Q-CAP), na lingua portuguesa do Brasil.

\section{MÉTODOS}

\section{Delineamento, população e ética}

Este é um estudo de construção e validação. O procedimento utilizado para construir e validar um instrumento depende de três fatores principais, a saber: 1) caracteristicas do instrumento de avaliação; 2) contextos da aplicação e 3) especificidades da populaçãoalvo [12]. Sendo assim, adotou-se, no presente estudo, um modelo que advém da combinação dos procedimentos propostos por quatro autores 
[6, 12-14]. Portanto, o processo de construção e validação do instrumento seguiu algumas etapas:

\section{Construção do instrumento}

Para a formulação do instrumento foi realizada uma ampla revisão de literatura sobre a asma e análise de conteúdo de alguns questionários existentes [9, 11, 15-19]. Assim, foi desenvolvido um instrumento composto por 20 itens que contemplam informações sobre tratamento, controle da asma e mitos populares sobre a doença.

\section{Estrutura do instrumento}

O Q-CAP destina-se aos pais, responsáveis legais e cuidadores de crianças e adolescentes com diagnóstico de asma. Sua aplicação é direta, podendo ser auto aplicado ou por entrevistador. Cada item deve ser lido como afirmação e as alternativas de resposta são: "Verdadeiro", "Falso" ou "Não sei". Todos os itens foram classificados em uma escala de Likert de três pontos, sendo atribuido 1 = Verdadeiro; 2 = Falso e 3 = Não sabe a resposta. Para sua codificação e avaliação final, os valores das alternativas corretas são transformados em um ponto e os valores incorretos, ou quando a resposta escolhida for "Não sabe", em zero, possuindo uma escala total de zero a 20 pontos. O ponto de corte será determinado pela avaliação da sensibilidade e especificidade através da Curva ROC (Receiver Operating Characteristic Curve) e classificado em "Niveis satisfatório" ou "Niveis insatisfatórios".

\section{Validade de construto e conteúdo}

Para fins de validação de construto, análise da consistência de conteúdo e referenciais relacionados[20], o questionário foi encaminhado à cinco pesquisadores especialistas da área, com ampla experiência clínica e produção bibliográfica. Foram realizados ajustes na estrutura das afirmações e categorias dos itens de acordo com as sugestões recebidas. Para fins de validação de conteúdo, foi realizado um estudo piloto, sendo aplicado o questionário em 30 pais de crianças com asma em atendimento ambulatorial. Tanto para validade de construto, quanto de conteúdo, os participantes deveriam conceituar as perguntas em três níveis ( $A, B$ e $C$ ), onde o conceito A corresponde a pergunta sem ajustes, B corresponde a pergunta com ajustes estruturais ou conceituais ou $\mathrm{C}$, correspondendo a perguntas com erros conceituais, devendo ser substituida.

\section{Propriedade psicométrica}

As propriedades psicométricas estudadas foram confiabilidade e reprodutibilidade, que avaliam se um instrumento é capaz de medir sempre da mesma maneira o que pretende medir, sendo consideradas cinco análises: a) consistência interna, mediante o coeficiente alfa de Cronbach, que avalia correlações específicas entre os itens do instrumento [21], ou seja, se um instrumento é capaz de mensurar sempre da mesma forma o que se pretende medir, realizando uma correlação média entre perguntas e respostas. Assim, o coeficiente $\alpha$ de Cronbach é calculado a partir da variância dos itens individuais e da variância da soma entre itens, verificando se todos utilizam a mesma escala de medição. Os valores foram considerados aceitáveis para pontuações de Coeficiente $\alpha$ de Cronbach >0,60; b) coeficiente de correlação intraclasse [22]: para mensurar a homogeneidade das medidas, servindo para interpretar a proporção da variabilidade total atribuida ao objeto medido, sendo adotado o ponto de corte $\geq 0,60$ para classificação do escore total (aceitável); c) Correlação de Pearson: aplicado a pontuação total entre o Q-CAP e o Newcastle Asthma Knowledge Questionnaire (NAKQ) para comparação da pontuação entre um instrumento previamente validado e a criação do Q-CAP [8]; d) Teste e reteste: para análise de concordância, foi aplicada a análise de Kappa, num periodo mínimo de 15 dias e máximo de 30 dias, sendo reavaliado os niveis de conhecimento em asma dos pais, sem nenhuma intervenção em educação da doença. Para avaliação do teste e reteste, foi selecionado um subgrupo de, ao menos, 20\% do total da amostra; e) Sensibilidade e especificidade: para fins de avaliação do ponto de corte, foi aplicado o coeficiente da Curva ROC para a pontuação total do Q-CAP e o ponto de corte do NAKQ em um subgrupo da amostra total. 


\section{Participantes da pesquisa}

A amostra é composta por pais de crianças com diagnóstico de asma em acompanhamento ambulatorial em um centro de referência em asma, localizado na cidade de Porto Alegre, região sul do Brasil. O centro possui uma equipe multidisciplinar e professores preceptores das Escolas da Pontificia Universidade Católica do Rio Grande do Sul, que supervisionam os alunos de graduação, pósgraduação e residência durante os atendimentos aos pacientes. No ambulatório selecionado, a equipe é composta por pneumologistas pediátricos, com ampla experiência clínica e produção bibliográfica sobre asma infantil. Em todas as consultas, os pais e reponsáveis recebem instruções teórico/práticas sobre a asma, tais como: características, sintomas, gravidade, efeitos dos medicamentos e técnicas inalatórias.

Os sujeitos foram selecionados na sala de espera do ambulatório, no dia agendado para a consulta médica regular com o pneumologista pediátrico, através de abordagem direta do entrevistador e explicação do estudo. Se mãe e/ou pai e/ou responsável concordasse em participar, era convidada a assinar o Termos de Consentimento Livre e Esclarecido. Após isso, eram aplicados os testes e os questionários.

Como critério de inclusão, os pais ou responsáveis deveriam possuir um periodo mínimo de seis meses de acompanhamento ambulatorial junto do paciente e residir com as crianças, mantendo contato direto entre criança/doença e tratamento, além de ter idade mínima de 18 anos. Como critério de exclusão, os pais não poderiam ser analfabetos ou possuir problemas cognitivos que pudessem influenciar nas análises de desfecho.

O estudo foi aprovado pelo Comitê de Ética em Pesquisa da Pontificia Universidade Católica do Rio Grande do Sul, via Plataforma Brasil (Parecer $n^{\circ}$ 3.092.959). Para a inclusão no estudo, foi solicitado que os participantes assinassem o
Termo de Consentimento Livre e Esclarecido, a fim de garantir a liberdade de participação nas entrevistas, a privacidade e o sigilo das informações recebidas.

\section{Coleta e análise dos dados}

Os dados foram coletados em uma tabela no banco de dados Microsoft Access versão 2013 e exportados para o software IBM SPSS Statistics 20 para Windows. Foram realizados os seguintes testes: coeficientes de alfa de Cronback e Coeficiente de correlação intraclasse, além da correlação de Pearson para fins de comparação entre dois instrumentos de avaliação (NAKQ e Q-CAP), análise de Kappa para fins de reprodutibilidade e sensibilidade e especificidade pelo coeficiente da curva ROC (NAKQ e Q-CAP). Além disso, os dados foram expressos por frequências absolutas e relativas para dados categóricos e média e desvio-padrão para dados contínuos.

No contexto da Teoria de Resposta ao Item é comumente utilizado a estimação ou calibração dos itens aonde avalia-se, para cada item, qual o valor de theta exigido para a obtenção de 50\%, fornecendo maior precisão aos escores dos testes. A calibração dos itens permite afirmar, que itens diferentes, terão resultados diferenciados, levando à maior fidedignidade da avaliação [23-25].

\section{RESULTADOS}

Participaram do estudo 132 sujeitos, sendo 117 $(88,6 \%)$ do sexo feminino, idade média de 35,9 $\pm 9,1$ anos, 78 (59,1\%) com ensino médio completo, 73 (59,8\%) pertencente a classe econômica C, sendo que $49(37,1 \%)$ relataram tabagismo residencial. Em relação aos pacientes, 108 (81,8\%) não estavam com a doença controlada, mesmo em acompanhamento ambulatorial especializado, conforme Tabela 1. Tanto os especialistas quanto os pais avaliados consideraram que o questionário abordava de forma objetiva os conceitos de asma, tinha validade de conteúdo e validade aparente adequadas. 
TABELA 1 - Caracteristicas gerais dos 132 pais e cuidadores dos pacientes asmáticos em acompanhamento ambulatorial pediátrico.

\begin{tabular}{|c|c|c|}
\hline Caracteristicas & $\mathbf{n}$ & $\%$ \\
\hline \multicolumn{3}{|l|}{ Raça } \\
\hline Branca & 73 & 55.3 \\
\hline Preta & 34 & 25,8 \\
\hline Parda & 25 & 18,9 \\
\hline \multicolumn{3}{|l|}{ Escolaridade (acompanhante) } \\
\hline Ensino básico & 13 & 9,8 \\
\hline Ensino fundamental & 38 & 28,8 \\
\hline Ensino médio & 78 & 59,1 \\
\hline Ensino superior & 3 & 2,3 \\
\hline \multicolumn{3}{|c|}{ Critério de classificação econômica* } \\
\hline Classe C & 79 & 59,8 \\
\hline Classe D & 52 & 39,4 \\
\hline Classe $\mathrm{E}$ & 1 & 0,8 \\
\hline \multicolumn{3}{|l|}{ Controle da asmat } \\
\hline Não controlada & 61 & 46,2 \\
\hline Parcialmente controlada & 47 & 35,6 \\
\hline Controlada & 24 & 18,2 \\
\hline \multicolumn{3}{|l|}{ Acompanhante } \\
\hline Pai & 15 & 11,4 \\
\hline Mãe & 108 & 81,8 \\
\hline Avós & 9 & 6,8 \\
\hline
\end{tabular}

*Associação Brasileira de Empresas de Pesquisa; HIniciativa Global Contra a Asma [1].
No geral, apenas cinco (3,8\%) pais demonstraram niveis satisfatórios de conhecimento em asma através da aplicação do Q-CAP. A média de pontuação foi de 7,5 $\pm 2,9$, dos 20 pontos totais do questionário. Um subgrupo (39 sujeitos) foi avaliado através do NAKQ resultando em 87,2\% (34) com pontuação abaixo dos níveis aceitáveis (pontuação de o a 31 , com ponto de corte aceitável em $\geq 21$ pontos),

Na Tabela 2 são apresentadas as questões da versão final do Questionário de Conhecimento em Asma Pediátrica (Q-CAP), juntamente com o gabarito das respostas. Esta versão foi utilizada no projeto piloto para os testes de validade de constructo e conteúdo, conforme descrito na metodologia, sendo que 30 pais responderam o questionário e atribuíram conceito "A" para ambos os testes em cada uma das questões. Já no que se refere aos valores do coeficiente de alfa de Cronbach por questões (itens), as pontuações máximas e mínimas tiveram uma variância entre 0,67 e 0,71 , respectivamente. Os escores totais do Q-CAP obtiveram conceito "A" nos testes de validade e valor de 0,69 no coeficiente alfa de Cronbach. Para fins de avaliação da correlação do coeficiente intraclasse, os resultados desmontaram que o questionário possui niveis dentro do aceitável (coeficiente de correlação intraclasse = 0,69; IC95\% = 0,61- 0,77; $\mathrm{p}<0,001)$. 
TABELA 2 - Questões, gabarito do Q-CAP, avaliação de construto ( $n=15)$, avaliação de conteúdo ( $n=15)$ e valor da consistência interna por alfa de Cronbach (por itens e escore total)

\section{Perguntas do questionário}

Gabarito alternativas
Teste de construto
Teste de conteúdo alfa de Cronbach
1 - A asma é uma deficiência nas defesas do organismo que facilita o surgimento de infecções respiratórias 2 - Os filhos têm mais chances de ter asma quando os pais são asmáticos

3 - A asma é uma doença emocional ou psicológica

4 - A alergia é um anticorpo que falta aos asmáticos

5 - O leite de vaca causa crises de asma ou piora a doença em algumas crianças

6 - Crianças com asma podem levar uma vida normal, sem restrição de suas atividades diárias

7 - Crianças com asma não devem praticar esportes que necessitam fazer muito esforço físico

8 - Possuir animal dentro de casa é proibido em familias de asmáticos

9 - O médico escutando os pulmões é suficiente para falar que a asma está controlada ou não 10 - Os antibióticos são parte importante do tratamento para asma

11 - O uso de corticoide oral em doses baixas/médias, nas crises recorrentes de asma, não faz mal para a saúde

12 - O tratamento com broncodilatador (salbutamol) faz mal para o coração

13 - A natação pode curar a asma

14 - A homeopatia pode curar a asma

15 - O corticoide inalatório tem a mesma função e indicação dos broncodilatadores

16 - Se a criança usar os remédios para a asma por muito tempo eles perdem o efeito

17 - Os remédios usados na "bombinha" podem viciar

18 - Os broncodilatadores são os melhores remédios preventivos para controlar a asma

19 - Doses baixas/médias de corticoide inalatório fazem mal a saúde quando usadas para tratar asma em crianças

20 - A morte por asma não pode ser prevenida

Escore total
F

V

F

F

F

V

F

F

F

F

V

F

F

F

F

F

F

F

F

F

$\geq 14^{*}$
A

A

A

A

A

A

A

A

A

A

A

A

0,67

A

A

A

A

0,70

A

A

0,68

A

A

0,67

0,69

* Ponto de corte 14 pontos (acertos), sendo: $\geq 14$ acertos = Niveis satisfatórios; $\leq 13$ acertos = Niveis insatisfatórios; Gabarito: V= verdadeira; F: falsa.

Q-CAP, Questionário de Conhecimento em Asma Pediátrica. 
Já para a análise de concordância foi aplicado o teste e reteste utilizando o Coeficiente de Kappa, em um subgrupo composto por 29 pais de crianças com asma. Dos 20 itens avaliados apenas o item 19 demonstrou valores abaixo dos niveis aceitáveis (Tabela $\mathbf{3}$ )

TABELA 3 - Avaliação do índice de Kappa (teste e reteste) do Q-CAP aplicado em 29 participantes, com intervalo de três a quatro semanas, sem aplicação de intervenção em asma.

\begin{tabular}{|c|c|c|c|c|c|c|}
\hline & \multicolumn{2}{|c|}{ Alternativa correta } & \multicolumn{2}{|c|}{ Alternativa incorreta } & \multirow{2}{*}{$\begin{array}{c}\text { Teste de } \\
\text { Kappa }\end{array}$} & \multirow{2}{*}{$p$} \\
\hline & Teste & Reteste & Teste & Reteste & & \\
\hline Questão 1 & 1 & 1 & 28 & 28 & 0,000 & 1,000 \\
\hline Questão 2 & 23 & 22 & 6 & 7 & 0,014 & 0.753 \\
\hline Questão 3 & 17 & 17 & 12 & 12 & 0,000 & 1,000 \\
\hline Questão 4 & 6 & 7 & 23 & 22 & 0,014 & 0,753 \\
\hline Questão 5 & 19 & 14 & 10 & 15 & 0,060 & 0,185 \\
\hline Questão 6 & 19 & 20 & 10 & 9 & $-0,013$ & 0,780 \\
\hline Questão 7 & 21 & 18 & 8 & 11 & 0,039 & 0,401 \\
\hline Questão 8 & 6 & 7 & 23 & 22 & $-0,010$ & 0,753 \\
\hline Questão 9 & 13 & 13 & 16 & 16 & 0,000 & 1,000 \\
\hline Questão 10 & 8 & 12 & 21 & 17 & $-0,042$ & 0,269 \\
\hline Questão 11 & 12 & 12 & 17 & 17 & 0,000 & 1,000 \\
\hline Questão 12 & 8 & 15 & 21 & 14 & $-0,075$ & 0,060 \\
\hline Questão 13 & 11 & 13 & 18 & 16 & $-0,022$ & 0,594 \\
\hline Questão 14 & 15 & 13 & 14 & 16 & 0,023 & 0,599 \\
\hline Questão 15 & 6 & 8 & 23 & 21 & $-0,020$ & 0.539 \\
\hline Questão 16 & 12 & 17 & 17 & 12 & $-0,057$ & 0,189 \\
\hline Questão 17 & 12 & 18 & 17 & 11 & $-0,070$ & 0,115 \\
\hline Questão 18 & 4 & 3 & 25 & 26 & 0,009 & 0,687 \\
\hline Questão 19 & 4 & 0 & 25 & 29 & 0,036 & $0,038^{*}$ \\
\hline Questão 20 & 10 & 8 & 19 & 21 & 0,020 & 0,570 \\
\hline
\end{tabular}

*: Teste de kappa com nível de significância de p<0,05. Q-CAP, Questionário de Conhecimento em Asma Pediátrica.

Uma sub amostra ( $n=39$ ) foi utilizada para calcular a Correlação de Pearson entre o Q-CAP e o NAKQ, tendo em vista ser um instrumento previamente validado, sendo que a avaliação da pontuação entre os questionários demonstrou haver uma correlação positiva de $r^{2}=0,525$ ( $p=$ 0,001) (Figura 1). 


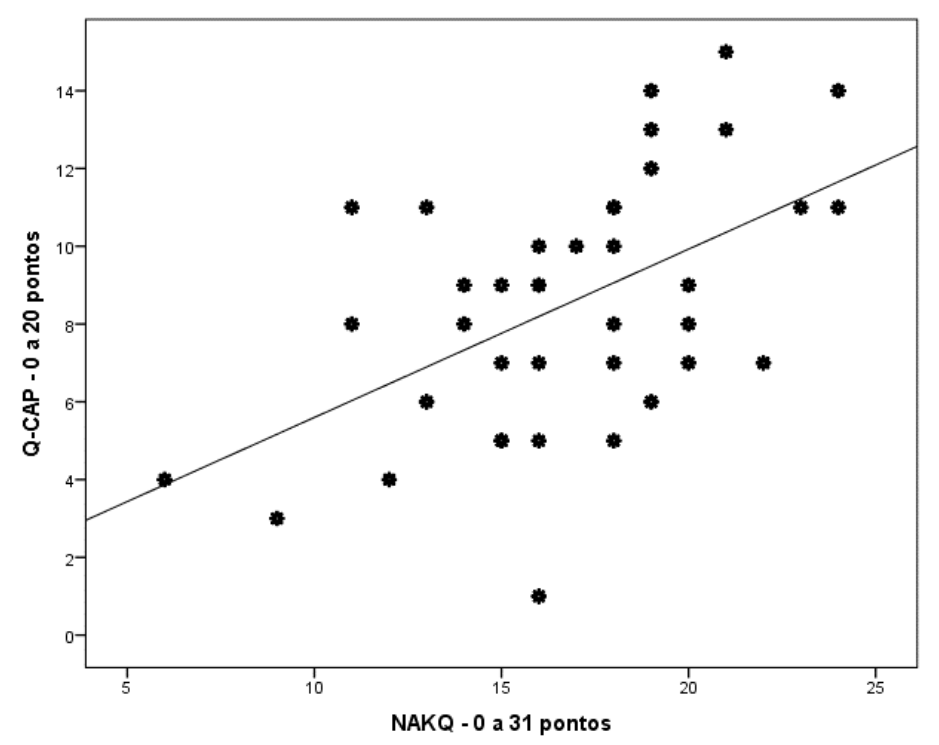

Figura 1 - Correlação de Pearson em uma sub amostra (n=39) para avaliação da pontuação entre o Q-CAP e o NAKQ, demonstrando haver uma correlação positiva de $\left(r^{2}=0,525 ; p=0,001\right)$. Q-CAP, Questionário de Conhecimento em Asma Pediátrica; NAKQ, Newcastle Asthma Knowledge Questionnaire.

Esta mesma sub amostra (39 sujeitos) avaliou a sensibilidade e especificidade do Q-CAP, tendo como referência a pontuação de corte do NAKQ. Os valores demonstraram que o Q-CAP possui ótima sensibilidade e especificidade, reportando uma área de 0,93 (IC95\%: 0,84 1,0; p=0,002). Para fins de ponto de corte, o Q-CAP atingiu valores de sensibilidade de $80 \%$ e especificidade de $97,1 \%$ (valor de $\geq 14$ pontos) (Figura 2).

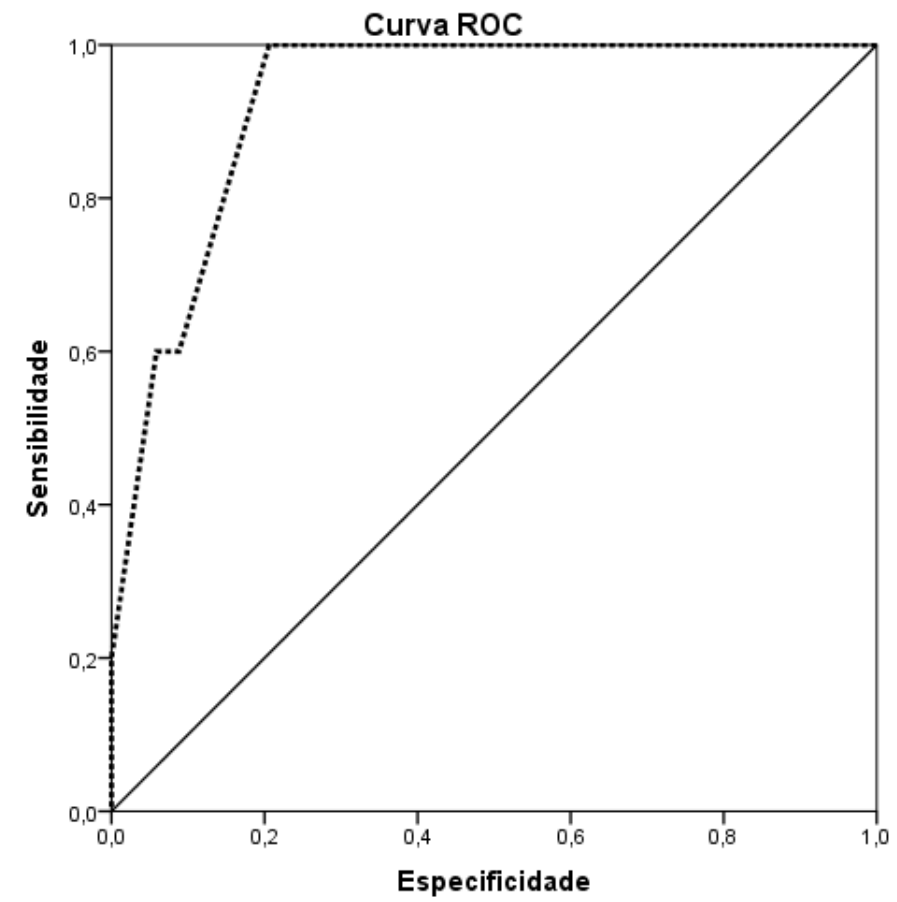

Figura 2 - Avaliação da sensibilidade e especificidade e ponto de corte do Q-CAP, tendo como base o ponto de corte do NAKQ. Q-CAP, Questionário de Conhecimento em Asma Pediátrica; NAKQ, Newcastle Asthma Knowledge Questionnaire. 


\section{DISCUSSÃO}

A educação em saúde tem se mostrado fundamental para o sucesso no tratamento de doenças crônicas como a asma, tanto em crianças quanto em adultos [26]. Nos pacientes pediátricos, os pais são os responsáveis pela gestão dos medicamentos e devem receber treinamento constante através dos médicos e programas de saúde pública. Sendo assim, o nivel de conhecimento acerca da doença deve ser avaliado sistematicamente, a fim de atestar as eventuais necessidades de correções conceituais. Entendendo dessa forma, nosso grupo desenvolveu e validou o Questionário de Conhecimento em Asma Pediátrica (Q-CAP), para os pais de crianças com diagnóstico de asma. Este é um questionário desenvolvido na lingua portuguesa-BR que levou em conta os aspectos culturais brasileiros, sem a necessidade de traduções ou adaptações, mostrando ser válido, confiável, de administração simples e acessivel para a população alvo.

Roncada et al. [7], encontraram, em sua revisão sistemática, 22 instrumentos especificos para avaliação do conhecimento em asma de pais e pacientes, sendo a maioria no idioma inglês e apenas um na lingua portuguesa do Brasil [27]. Porém, os critérios de qualificação desses artigos, em geral, ficaram com baixa avaliação positiva ou intermediária, principalmente nos itens de validade de constructo, consistência interna e reprodutibilidade. Para Hair Junior et al. [28], a confiabilidade é indicada pela consistência na mensuração dos indicadores de uma variável latente e a ela, é atribuida um grau ou conceito. Neste sentido, a análise do Q-CAP demonstrou ser um instrumento confiável, pois os testes de constructo e validade obtiveram conceito " $\mathrm{A}$ ", bem como os valores da consistência interna, que alcançaram resultados adequados, confirmando que o instrumento realmente infere ou mede aquilo que se propõe com qualidade.

O NAKQ foi desenvolvido por Fitzclarence et al. para avaliar o conhecimento dos pais de crianças com asma [9]. Em 2016, nosso grupo realizou a sua validação linguística e psicométrica para o idioma português do Brasil e, posteriormente, para a população de pais de crianças asmáticas(8) [8, 11]. Por se tratar de um instrumento reconhecido internacionalmente e estarmos apropriados de suas características, o elegemos para realizar a comparação entre as pontuações encontradas nele e no Q-CAP (Q-CAP vs. NAKQ). Em ambos os questionários as pontuações ficaram abaixo dos níveis satisfatórios de conhecimento sobre asma, sendo que menos de $13 \%$ dos pais atingiram escores totais acima dos pontos de corte. Resultados semelhantes já haviam sido encontrados em estudos anteriores [8, 29], sugerindo que as políticas públicas de educação em saúde ainda são ineficazes em países em desenvolvimento. No Brasil, as ações de educação em asma devem ser expandidas, também, para médicos pediatras e outros membros da equipe, tendo em vista muitos destes não possuírem niveis satisfatórios de conhecimento teórico/ prático do manejo da doença [30].

Em relação à análise das respostas através do Coeficiente de Pearson houve uma correlação positiva significativa entre eles, o que possibilita considerar O Q-CAP um instrumento com potencial internacional.

Para determinar o ponto de corte adequado do questionário, foi avaliada a sensibilidade e especificidade tendo como base o ponto de corte do NAKQ. Este teste é fundamental para ajustar o limite desejável entre o conhecimento satisfatório, ou insatisfatório, dos sujeitos objetos da aplicação do Q-CAP. Pode-se verificar que a sensibilidade alcançou valores de $80 \%$, desmontando grande capacidade de identificar corretamente aqueles individuos que realmente possuem conhecimento adequado sobre a doença. Já a especificidade, que é a capacidade do teste de identificar corretamente os indivíduos que não possuem o conhecimento satisfatório, resultou em valores de 97,1\%. Assim, podemos afirmar que o ponto de corte (14 pontos) encontrado representa a quantidade mínima de conhecimento que os pais de crianças com asma devem possuir para propiciar a gestão adequada do tratamento do seu filho.

Com a construção e validação do Q-CAP 
acreditamos que inúmeros pacientes serão beneficiados no Brasil, pois terão um instrumento que foi desenvolvido observando os aspectos regionais, sem adaptações de termos e conceitos estrangeiros distantes da nossa realidade. Neste estudo, pode-se observar estes beneficios, pois o Q-CAP apresentou niveis satisfatório em apenas 3,8\% dos pais, enquanto o percentual do NAKQ foi de 12,8\%. Esta diferença corrobora com os resultados das análises subjetivas realizadas pela equipe de saúde durante as consultas, os quais demonstraram quantitativos inferiores a $7 \%$ de pais com niveis satisfatórios de conhecimento em asma. Já para os profissionais da saúde, o uso do Q-CAP vai permitir mensurar o conhecimento dos pais de forma rápida e objetiva, dando oportunidade para a customização do ensino, observando as limitações e necessidades de cada indivíduo, resultado em maior adesão ao tratamento e melhora na qualidade de vida do paciente e familiares.

Sendo assim, o Q-CAP demonstrou ser um instrumento confiável e com adequados indices de reprodutibilidade e consistência interna. Sua aplicação é fácil e abrange os aspectos culturais brasileiros essenciais, além de grande precisão em mensurar o nivel de conhecimento sobre asma. Não somente a população brasileira, mas outros paises de língua portuguesa poderão ser beneficiados por esse trabalho.

\section{REFERÊNCIAS}

1. Boulet LP, FitzGerald JM, Reddel HK. The revised 2014 GINA strategy report: opportunities for change. Curr Opin Pulm Med. 2015;21(1):1-7.

2. Cardoso TA, Roncada C, Silva ERD, Pinto LA, Jones MH, Stein RT, Pitrez PM. The impact of asthma in Brazil: a longitudinal analysis of data from a Brazilian national database system. J Bras Pneumol. 2017:43(3):163-8. doi: 10.1590/S180637562016000000352 .

3. Roncada C, de Oliveira SG, Cidade SF, Sarria EE, Mattiello R, Ojeda BS, Dos Santos BR, Gustavo Ada S, Pinto LA, Jones MH, Stein RT, Pitrez PM. Burden of asthma among inner-city children from Southern Brazil. J Asthma. 2016;53(5):498-504. doi: 10.3109/02770903.2015.1108438.
4. Guevara JP, Wolf FM, Grum CM, Clark NM. Effects of educational interventions for self management of asthma in children and adolescents: systematic review and meta-analysis. BMJ. 2003:326(7402):1308-9.

5. Roncada C, Oliveira SG, Cidade SF, Rafael JG, Ojeda BS, Santos BR, Gustavo Ada S, Pitrez PM. Mitos populares e características do tratamento da asma em crianças e adolescentes de zona urbana do sul do Brasil. J Bras Pneumol. 2016;42(2):136-42. doi: 10.1590/S1806-37562015000000166

6. Terwee CB, Bot SD, de Boer MR, van der Windt DA, Knol DL, Dekker J, Bouter LM, de Vet HC. Quality criteria were proposed for measurement properties of health status questionnaires. J Clin Epidemiol. 2007:60(1):34-42. doi: 10.1016/j.jclinepi. 2006.03.012

7. Roncada C, Dias CP, Sarria EE, Pitrez PM. Instrumentos especificos para avaliação do conhecimento em asma: revisão sistemática. Medicina (Ribeirao Preto Online). 2015;48(6):619-27.

8. Roncada C, Bischoff LC, Bugança BM, Soldera $\mathrm{K}$, de Araujo Cardoso T, Pitrez PM. Características psicométricas do Questionário Newcastle de Conhecimento em Asma (NAKQ) para pais de crianças com asma. Sci Med. 2017;27(2):2

9. Fitzclarence CA, Henry RL. Validation of an asthma knowledge questionnaire. J Paediatr Child Health. 1990;26(4):200-4

10. Wigal JK, Stout C, Brandon M, Winder JA, McConnaughy $\mathrm{K}$, Creer TL, Kotses H. The Knowledge, Attitude, and Self-Efficacy Asthma Questionnaire. Chest. 1993:104(4):1144-8. doi: 10.1378/chest.104.4.1144.

11. Cidade SF, Roncada C, Costa DD, Rafael JG, Pitrez PM. Validação linguística e psicométrica do questionário sobre conhecimento em asma Newcastle Asthma Knowledge Questionnaire. Rev Ciênc Méd. 2015;24(2):45-54.

12. Borsa JC, Damásio BF, Bandeira DR. Adaptação e validação de instrumentos psicológicos entre culturas: algumas considerações. Paidéia. 2012;22(53):423-32.

13. Gjersing L, Caplehorn JR, Clausen T. Cross-cultural adaptation of research instruments: language, setting, time and statistical considerations. BMC Med Res Methodol. 2010;10(1):13.

14. Pasquali L. Instrumentação Psicológica: Fundamentos E Práticas. Porto Alegre: Artmed;2010.

15. Cabelloa M, Oceja-Setienb E, Higueraa LG, Caberoa M, Belmontea EP, Gómez-acebob I. Assessment of parental asthma knowledge with the Newcastle Asthma Knowledge Questionnaire. Rev Pediatr. 2013:15:117-26.

16. Contreras-Fernandez E, Baron-Lopez FJ, Mendez-Martinez C, Canca-Sanchez JC, Cabezon Rodriguez I, Rivas-Ruiz F. Validation of the knowledge and attitudes of health professionals in the Living Will Declaration process. Aten Primaria. 2017;49(4):233-9. 
17. Fadzil A, Norzila MZ. Parental asthma knowledge. Med J Malaysia. 2002:57(4):474-81.

18. Federman AD, Wolf M, Sofianou A, Wilson EA, Martynenko M, Halm EA, Leventhal H, Wisnivesky JP. The association of health literacy with illness and medication beliefs among older adults with asthma. Patient Educ Couns. 2013:92(2):273-8. doi: 10.1016/j. pec.2013.02.013. Epub 2013 Mar 21.

19. Roncada C, Oliveira SG, Cidade SF, Rafael JG, Ojeda BS, Santos BR, Gustavo Ada S, Pitrez PM. Asthma treatment in children and adolescents in an urban area in southern Brazil: popular myths and features. J Bras Pneumol. 2016;42(2):136-42. doi: 10.1590/S1806-37562015000000166.

20. Alexandre NMC, Coluci MZO. Validade de conteúdo nos processos de construção e adaptação de instrumentos de medidas. Ciência \& Saúde Col. 2011:16(7):3061-8.

21. Cronbach LJ. Coefficient alpha and the internal structure of tests. Psychometrika. 1951;16(3):297-334.

22. Bartko JJ. The intraclass correlation coefficient as a measure of reliability. Psychological reports. 1966;19(1):3-11

23. da Silva Nunes CHS, Primi R. Impacto do tamanho da amostra na calibração de itens e estimativa de escores por teoria de resposta ao item. Avaliaçõo Psicológica. 2005:4(2):141-53.

24. Andrade JMd, Laros JA, Gouveia VV. O uso da teoria de resposta ao item em avaliações educacionais: diretrizes para pesquisadores. Avaliação Psicológica. 2010;9(3):421-35.

25. Thomas ML. The value of item response theory in clinical assessment: a review. Assessment. 2011:18(3):291-307.

26. Williams MV, Baker DW. Honig EG, Lee TM, Nowlan A. Inadequate literacy is a barrier to asthma knowledge and self-care. Chest. 1998;114(4):1008-15.

27. Borges MC, Ferraz E, Pontes SM, Cetlin Ade C, Caldeira RD, Silva CS, Araújo AC, Vianna EO. Desenvolvimento e validação de um questionário de conhecimento em asma para uso no Brasil. J Bras Pneumol. 2010;36(1):8-13. doi: 10.1590/S180637132010000100004

28. Hair JF, Black WC, Babin BJ, Anderson RE, Tatham RL. Análise multivariada de dados. Porto Alegre: Bookman. 2009.

29. Kuti BP, Omole KO. Factors associated with caregivers' knowledge about childhood asthma in llesa, Nigeria. Ann Nigerian Med. 2016;10(1):30-6.

30. Cordeiro NGB, Cunha AJLA, Kuschnir FC. Conhecimento sobre asma de pediatras de hospitais públicos do Rio de Janeiro. Arq Asma Alerg Imunol. 2018;2(1):108-15
NOTAS

\section{Apoio financeiro}

Este estudo não recebeu apoio financeiro de fontes externas.

\section{Declaração de conflito de interesses}

Os autores declaram não haver conflitos de interesses relevantes ao conteúdo deste estudo.

\section{Contribuições dos autores}

Todos os autores fizeram contribuições substanciais para concepção, ou delineamento, ou aquisição, ou análise ou interpretação de dados; e redação do trabalho ou revisão crítica; e aprovação final da versão para publicação.

\section{Disponibilidade dos dados e responsabilidade pelos resultados}

Todos os autores declaram ter tido total acesso aos dados obtidos e assumem completa responsabilidade pela integridade destes resultados.

\section{Correspondência}

Thiago de Araujo Cardoso

Rua Vinte e Quatro de Maio, 141 - Centro, Osório - RS - 95520-000.

Instituição: Centro Universitário Cenecista de Osório (UNICNEC), Curso de Educação Física 